\section{A home tank aquatic shuttlebox}

\author{
FRANK ETSCORN* \\ Western Kentucky University \\ Bowling Green, Kentucky 42101
}

The typical construction of aquatic shuttleboxes currently in experimental use generally requires transferring the fish from its home tank to the training apparatus. The aquatic shuttlebox to be described here was expressly designed to eliminate any need for removing the $\mathrm{S}$ from water or its home tank (i.e., housing and training is in the same tank).

As can be seen in Fig. 1, the shuttlebox is simply lowered into and onto the home tank, with little, if any, disruption of ongoing behavior. Outside dimensions of the shuttlebox measure $5 \mathrm{~W} \mathrm{x} \mathrm{43/4} \mathrm{D} \times 9.3 / 8$ in. L. Of course, these figures would vary according to the length of fish used (the device pictured is for fish $2-3$ in. long). Electrodes for delivering the noxious (shock) stimulus were fashioned from stainless steel rods $51 / 4 \times 1 / 4$ in., with $1 \frac{1}{2}$ in. of threading to allow for vertical adjustments. When used with a "TV-Tank" (made by Sternco Plastics and sold for approximately 70 cents), the shuttlebox can be adjusted to "lock in" with the sides of the tank, thus eliminating any variation in its position from trial to trial or $S$ to $S$. By removing two small bolts, the central barrier can easily be removed and replaced with various swim-through targets (circles, squares, or triangles) or replaced with swim-under or swim-over hurdles. Likewise, the flat black aluminum end panels can be removed and replaced with end panels drilled to accept multiple cue lights (since the TV-Tank is clear, various colors can be used). During training sessions when multiple shuttleboxes are used in close proximity of each other, it is important to avoid the confounding effects of the cue lights from nearby shuttleboxes. This potential problem was remedied by bolting on flat black aluminum side panels; thus, in conjunction with the end panels, fully enclosing the device from extraneous light sources. (In studies using this device, we have usually mounted small incandescent lamps over both sides of the central barrier in order to allow sufficient light for the fish to orient itself within the tank.) The side panels may also be fitted with photoelectric cells to sense each response under a hurdle or through a target.

Construction details may be obtained from the author.

(Received for publication August 18, 1973; revision received October 4,1973 .)

\footnotetext{
*Present address: George Peabody College for Teachers, Department of Psychology, 21st Avenue South, Nashville, Tennessee 37203 .
}

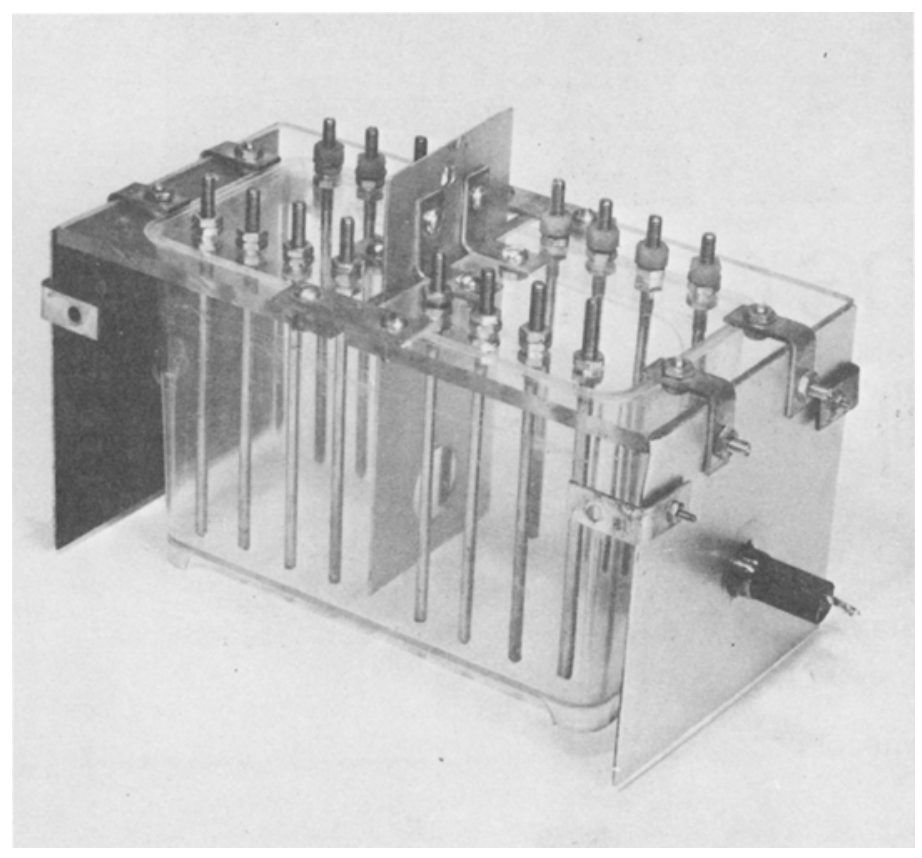

Fig. 1. 